\title{
The Phonetic Nature of Consonants in Modern Standard Arabic
}

\author{
Mohammad Yahya Bani Salameh ${ }^{1}$ \\ ${ }^{1}$ Tabuk University, Saudi Arabia \\ Correspondence: Mohammad Yahya Bani Salameh, Tabuk University, Saudi Arabia. Tel: 966-58-0323-239. E-mail: \\ drms78@yahoo.com
}

Received: June 29, 2015

Accepted: July 29, 2015

Online Published: August 5, 2015

doi:10.5430/elr.v4n3p30

URL: http://dx.doi.org/10.5430/elr.v4n3p30

\begin{abstract}
The aim of this paper is to discuss the phonetic nature of Arabic consonants in Modern Standard Arabic (MSA). Although Arabic is a Semitic language, the speech sound system of Arabic is very comprehensive. Data used for this study were collocated from the standard speech of nine informants who are native speakers of Arabic. The researcher used himself as informant, He also benefited from three other Jordanians and four educated Yemenis. Considering the alphabets as the written symbols used for transcribing the phones of actual pronunciation, it was found that the pronunciation of many Arabic sounds has gradually changed from the standard. The study also discussed several related issues including: Phonetic Description of Arabic consonants, classification of Arabic consonants, types of Arabic consonants and distribution of Arabic consonants.
\end{abstract}

Keywords: Modern Standard Arabic (MSA), Arabic consonants, Dialectal variation, Consonants distribution, Consonants classification.

\section{Introduction}

The Arabic language is one of the most important languages of the world. With it is growing importance of Arab world in the International affairs, the importance of Arabic language has reached to the greater heights. Since the holy book Qura' $n$ is written in Arabic, the language has a place of special prestige in all Muslim societies, and therefore more and more Muslims and Asia, central Asia, and Africa are learning the Arabic language, the language of their faith. Moreover there is a large number of people around the globe who learn this language because of its political importance. The importance of Arabic has also increased because of the prestigious position of the Arab countries in the world of international trade and commerce.( Fatihi, A.R. 2001)

\subsection{Afro-Asiatic Languages}

Arabic belongs to the Afro-Asiatic Languages. The Afro-Asiatic is a tie family of languages covers most of Africa, north of Sahara and the Sahara itself dipping down on the eastern side of the continent. The Afro-Asiatic family of language is also a major language family of South Western Asia. The family has five branches: Semitic group of languages, Egyptian, Berber, Cushitic and Chadic. After serving for millennia as the language of one of the world's most spectacular civilizations, Egyptian family of languages, comprising ligypiian, the language of Ancient Egypt, has been extinct. The Cushilic and Chadic languages are sufficiently different from each other. Thus the Semitic family of languages is the most dominating linguistic family among all the-Afro-Asiatic languages.( Fatihi, A.R. 2001)

\subsection{Semitic Family of Languages}

Arabic is one of the major languages of Semitic family. To be more specific it is an off-shoot of the languages of south west Asia. The name Semitic owes its origin to SHCM, mentioned in chapter X of Book of Genesis. The chapter deals with ARAM, ASHER, and CBER; that is Ramites, Assyrians, and Hebrew. The scholars of theology and history name these groups of people Semitic Races. Their relation with each other is evident by the close similarity and strong connection among them. Subsequent excavations have brought to light new information about some more races having similar characteristics. In the term Semitic has acquired a wider sense. Arabic belongs to the southern group of Semitic languages; this group includes the ancient Southern Arabic, with different dialects namely SABAEAN, MINAEAN, QATABANJAN, and HADRAMAWTEAN. They go back to the period extending over $8^{\text {th }}$ century B.C. 10 full century A, D).

The oldest texts in classical Arabic are found in the inscription of ZABAD (512 A.D.), inscription of HARRAN (568 A.D.) and inscription of UMMUL JIMAL (568 A D.). This lexis alone cannot explain the sublimity of the classical 
Arabic as evidenced in the Pre-Islamic Arabic poetry. By the sixth century A.D. the classical language of the pre-Islamic poetry had attained a stage of perfection, so much so that it could receive the inimitable Arabic of the holy book AL QURAAN. In the $8^{\text {th }}$ century A. D. the scholars like AN Khalil and Sibawahi got interested in the development of the language and presented their observations in pioneering works like Al-Kitab which is supposed to be one of the greatest monuments of human intelligence.

\subsection{Dialectal Variation}

It can be said that Arabic is one of the most important languages of the modern world. A full understanding of the structure of the language may help in establishing the dialectal variation of Arabic. The central concept underlying the term dialect lies in the local variations in pronunciation, grammar or vocabulary. Though many criteria have been used for determining the status of a dialect within a speech community, yet most of the linguist tends to concentrate on the criterion of 'mutual intelligibility'. From the point of mutual intelligibility all the regional styles of Arabic can be viewed from two spatial dimensions, i.e., lying next to one another and spoken at each end of the language area. In both these situations there is hardly any problem of mutual intelligibility at any level of linguistic expression. (Chejne,A.1958)

The phonetic peculiarities of different formal styles of pronunciation, including the permissible degree of reduction and assimilation of speech sounds, are also an important criterion for designating a particular speech as regional variation from other regional styles of the same language. Regional variations in Arabic may be assigned to geographical factors, which had made inter-communication difficult among different regions of Arab world in the past. Consequently each region along with many other social and ethnic factors developed its own peculiarities exhibited at different levels of the variety spoken in that area. Moreover, the language contact and inter dialectal borrowings, too have contributed to dialectal variations. In Arabic clearer dialectal divisions are obtainable in the areas where mutual intercourse among the people living in different areas is hindered due to physical barriers such as high mountain ranges or wide rivers.(Greenberg, J. 1978)

At present, however, the increasing contact brought in by developed systems of communications, education and urbanization, among the speakers of different dialects of Arabic has greatly affected the speech habits of different regions and consequently, has made the task of demarcation of various dialects very difficult, by minimizing dialectal differences to a greater extent.

\section{Arabic Phonology}

The speech sound system of Arabic is very comprehensive. Considering the alphabets as the written symbols used for transcribing the phones of actual pronunciation, it has adequately covered the maximum number of sounds available in other languages. The human vocal system of articulator apparatus is a complete musical device and the elegance of this system has manifested to the fullest extent in the Arabic Phonology. Thus there are some sounds which are exclusively found only in Arabic Phonological system and not in any other language, such as /D/,/DH/,/S/,/T/, although we may come across some similar sounds there, but these are not well defined and distinguished from the aspect of articulation. If we consider that he primary task of phonology is to provide objective description of speech, then the sound symbols or notational symbols of Arabic phonology are efficient description of possible variety of articulation and sound patterns.

Keeping in view the arbitrary nature of linguistics, one remarkable fact about pronunciation is that it is in continuous process of alteration generation after generation. This is a process of alteration generation after generating. This process is natural and many factors, ranging from individual psychological and social factors play their role in the change. This is a universal law of nature; therefore, no phonological system of any language is immune to change.

Speech sounds are perpetual target of change and variation. The process involves many factors which have implosive and arbitrary nature. It is to be observed in principle that to determine the trends of this variation is not an easy job, because the process seems to be such slow and complex and takes a long natural course before it attracts the attention of observers and researchers. Departing from this point, we notice that the pronunciation of many Arabic sounds has gradually changed from the standard one in many colloquial dialects.

Apart from these minor developments in some regional dialects of Arabic language, the phonological base of the standard Arabic defined and resisted any change. This is exceptional in case of Arabic language, as no other language ever could maintain the consistency of its phonological sequences. 


\section{Definition of Modern Standard Arabic (MSA)}

A fully agreed - upon of MSA does not yet exist, but there is a general consensus that modern Arabic writing in all its forms constitutes the basis of the identity of the language. Modern writing, however, covers an extensive range of discourse styles and genres ranging from complex and conservative to innovative and experimental. Finding a standard that is an identifiable segment of the modern Arabic writing language used for media purposes, and it has been the focus of linguists' attention for a number of years because its stability, its pervasiveness, and its ability to serve as a model of contemporary written usage. Dissemination of a written (and broadcast) prestige standard by the news media is a wide-spread phenomenon, especially in multilingual, diglossic, and multi-dialectal societies.

Elsaid Badawi's phrase fuSHâ al-âsr (فصحى العصر) is his Arabic term for MSA (1985, p.17), which he locates on a continuum (at "level two").

As he points out, the levels "are not segregated entities", $(1985$, p.17) but shade into other levels gradually. He identifies level two (MSA) as "mostly written" rather than spoken, and levels two and three as essentially "in complementary distribution" with each other $(1985$, p.19) that is, they function separate spheres, with some overlap. (Karin, C. Ryding. 2006)

\section{Purpose}

The purpose of this study is to highlight the consonantal system in Modern Standard Arabic (MSA). The study further delves into the phonological nature of Arabic consonants with regard to this particular variety, discussing several related issues including: Phonetic Description of Arabic consonants, classification of Arabic consonants s, types of Arabic consonants and distribution of Arabic consonants.

\section{Methodology}

Data used for this study were collocated from the standard speech of eight informants who are native speakers of Arabic. The researcher used himself as informant, he also benefited from four educated Yemens and three Jordanians whose Linguistic behaviour is judged to be /faSi:H/ 'clear', 'eloquent' through several successive sittings. These four Yemenis and three Jordanians, they are as follows:

$$
\begin{aligned}
& \text { Four Ph.D scholars. (Yemenis) } \\
& \text { Three MA students in AMU (Jordanians) }
\end{aligned}
$$

\section{Introducing Consonantal System in Arabic:}

Arabic language consists of twenty-eight consonants which are categorized into stops, nasals, laterals trills, fricatives, affricates, and semivowels. Some peculiar velarized phonemes, also known as emphatics, form a fundamental part of Arabic consonantal system.

\section{Phonemic-Contrast}

The classic case of contrast, which is central to the phonemic principle, is the minimal pair phenomenon:

If two words differ as to only one sound, then the distinction between the two sounds must be phonemic. (Philip) Carr, 1993).

\subsection{Consonant-contrast}

Minimal pair

\begin{tabular}{|l|l|l|l|}
\hline No. & Symbol & Arabic Example & English Meaning \\
\hline 1. & $/ ' /$ & /qara'a/ & 'to read' \\
\hline 2. & $/ ` /$ & $/$ qara'/ & 'he knocked' \\
\hline 3. & $/ \mathrm{b} /$ & $/ \mathrm{bard} /$ & 'coldness' \\
\hline 4. & $/ \mathrm{f} /$ & $/ \mathrm{fard} /$ & 'individual' \\
\hline 5. & $/ \mathrm{n} /$ & $/$ nakara/ & 'he denied' \\
\hline 6. & $/ \mathrm{m} /$ & $/$ makara/ & 'he deceived' \\
\hline 7. & $/ \check{\mathbf{s}} /$ & /saraha/ & 'he explained' \\
\hline 8. & $/ \mathrm{s} /$ & /saraHa/ & 'he went astray' \\
\hline
\end{tabular}




\begin{tabular}{|c|c|c|c|}
\hline 9. & $\mid \mathrm{z} /$ & /zara'a/ & 'he cultivated' \\
\hline 10. & $/ \mathrm{S} /$ & /Sara“/ & 'he throw s.o-'down' \\
\hline 11. & $/ \mathrm{d} /$ & /di:n/ & 'religion' \\
\hline 12. & 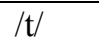 & $/ \mathrm{ti}: \mathrm{n} /$ & 'fig' \\
\hline 13. & $/ \mathrm{T} /$ & /Ti:n/ & 'clay' \\
\hline 14. & $/ \underline{\mathrm{D}} /$ & /Daba/ & 'to be melted' \\
\hline 15. & $/ \theta /$ & /Oaba/ & 'to regain onsciousness, reward' \\
\hline 16. & $/ \mathrm{r} /$ & /qar"/ & 'knocking', rapping, beating, thumping' \\
\hline 17. & $/ 1 /$ & $/ \mathrm{qal}^{\prime} /$ & 'rooting out' \\
\hline 18. & $/ \mathrm{h} /$ & /hazama/ & 'he defeated' \\
\hline 19. & /H/ & /Hazama/ & 'he tied-up', bundled \\
\hline 20. & $/ \breve{\mathrm{g}} /$ & /ğazama/ & 'he cut off' to judge, decide \\
\hline 21. & $/ \mathrm{x} /$ & /xasafa/ & 'to be eclipsed' (moon) \\
\hline 22. & $/ \mathrm{k} /$ & /kasafa/ & 'tobe eclipsed' (sun) \\
\hline 23. & $/ \mathrm{q} /$ & qalaba & 'he returned around', 'turn over' \\
\hline 24. & /G/ & /Galaba/ & 'he defeated, 'to overcome' \\
\hline 25. & $/ \mathrm{D} /$ & /naDir/ & 'flourshing, fresh, verdant' \\
\hline 26. & $/ \mathrm{DH} /$ & /naDHir/ & 'similar, equal, an equivalent' \\
\hline 27. & $/ \mathrm{w} /$ & /'a:wana/ & 'he helped' \\
\hline 28. & $/ \mathrm{y} /$ & /'a:yana/ & 'he inspected, examined' \\
\hline
\end{tabular}

Table 1

In Arabic there are 28 consonants (or consonantal phonemes, to be precise). These are listed below along with examples that illustrate their occurrence in the initial, medial and final positions.

7.2 Classification based on place of articulation:

\begin{tabular}{|l|l|}
\hline Phonetic Description & Symbol \\
\hline bilabial (3) & /b, m, w/ \\
\hline labio-dental(1) & /f/ \\
\hline dental (4) & /d,t, D, T/ \\
\hline inter-dental(3) & $/ \theta, \underline{D}, \mathrm{DH} /$ \\
\hline alveolar(6) & $/ \mathrm{s}, \mathrm{z}, \mathrm{S}, \mathrm{n}, \mathrm{r}, \mathrm{l} /$ \\
\hline Palato-alveolar (2) & $/ \mathrm{s}, \mathrm{y} /$ \\
\hline palatal (1) & /y/ \\
\hline velar (2) & $/ \mathrm{k}, \breve{\mathrm{g}} /$ \\
\hline uvular (2) & $/ \mathrm{q}, \mathrm{x}, \mathrm{G} /$ \\
\hline pharyngeal (2) & $/ \mathrm{H}, \% /$ \\
\hline glottal (2) & $/ \mathrm{h}, \% /$ \\
\hline
\end{tabular}

Table 1 
7.3 Classification base on manner of articulation:

\begin{tabular}{|l|l|}
\hline Phonetic Description & Symbol \\
\hline Stops(9) & \multicolumn{1}{c|}{$/ \mathrm{b}, \mathrm{t}, \mathrm{T}, \mathrm{d}, \mathrm{D}, \mathrm{k}, \mathrm{q}, \mathrm{h},{ }^{\prime} /$} \\
\hline Nasals(2) & $/ \mathrm{l} /$ \\
\hline Lateral(1) & $/ \mathrm{r} /$ \\
\hline Trill(1) & $/ \mathrm{f}, \mathrm{\theta}, \underline{\mathrm{D}}, \mathrm{DH}, \mathrm{s}, \mathrm{S}, \mathrm{z}, \breve{\mathrm{s}}, \mathrm{x}, \mathrm{h}, \mathrm{G}$, / Affricate $(1)$ \\
\hline Fricatives(12) & $/ \mathrm{g} /$ \\
\hline Semivowels(2) & $/ \mathrm{w}, \mathrm{y} /$ \\
\hline
\end{tabular}

Table 1

List of transcription system of Modern Standard Arabic (MSA)

\begin{tabular}{|c|c|}
\hline (MSA) consonants & IPA \\
\hline $\mathrm{b}$ & $\mathrm{B}$ \\
\hline $\mathrm{t}$ & $\mathrm{T}$ \\
\hline $\mathrm{d}$ & $\mathrm{D}$ \\
\hline $\mathrm{T}$ & $\underline{\mathrm{T}}$ \\
\hline $\mathrm{D}$ & $\underline{\mathrm{D}}$ \\
\hline $\mathrm{k}$ & $\mathrm{K}$ \\
\hline q & Q \\
\hline , & $?$ \\
\hline $\mathrm{h}$ & $\mathrm{h}$ \\
\hline$\breve{\mathrm{g}}$ & $\mathrm{j}$ \\
\hline$f$ & $\mathrm{f}$ \\
\hline$\theta$ & $\theta$ \\
\hline $\mathrm{S}$ & $\mathrm{s}$ \\
\hline $\mathrm{S}$ & $\underline{\mathrm{S}}$ \\
\hline $\mathrm{x}$ & $\mathrm{x}$ \\
\hline$\underline{\mathrm{D}}$ & ð \\
\hline $\mathrm{DH}$ & ð \\
\hline $\mathrm{z}$ & $\mathrm{z}$ \\
\hline \multicolumn{2}{|l|}{$\mathrm{G}$} \\
\hline ' & $\varepsilon$ \\
\hline $\mathrm{m}$ & $\mathrm{m}$ \\
\hline $\mathrm{n}$ & $\mathrm{n}$ \\
\hline 1 & 1 \\
\hline r & $\mathrm{r}$ \\
\hline $\mathrm{W}$ & $\mathrm{W}$ \\
\hline $\mathrm{y}$ & $\mathrm{y}$ \\
\hline
\end{tabular}

Table 2

The above transcription system is illustrated of the following table reproduced from Fatihi, A.R. (2001, p. 69) “Aspects of Arabic Phonology". Kotinga Publications, Delhi. 


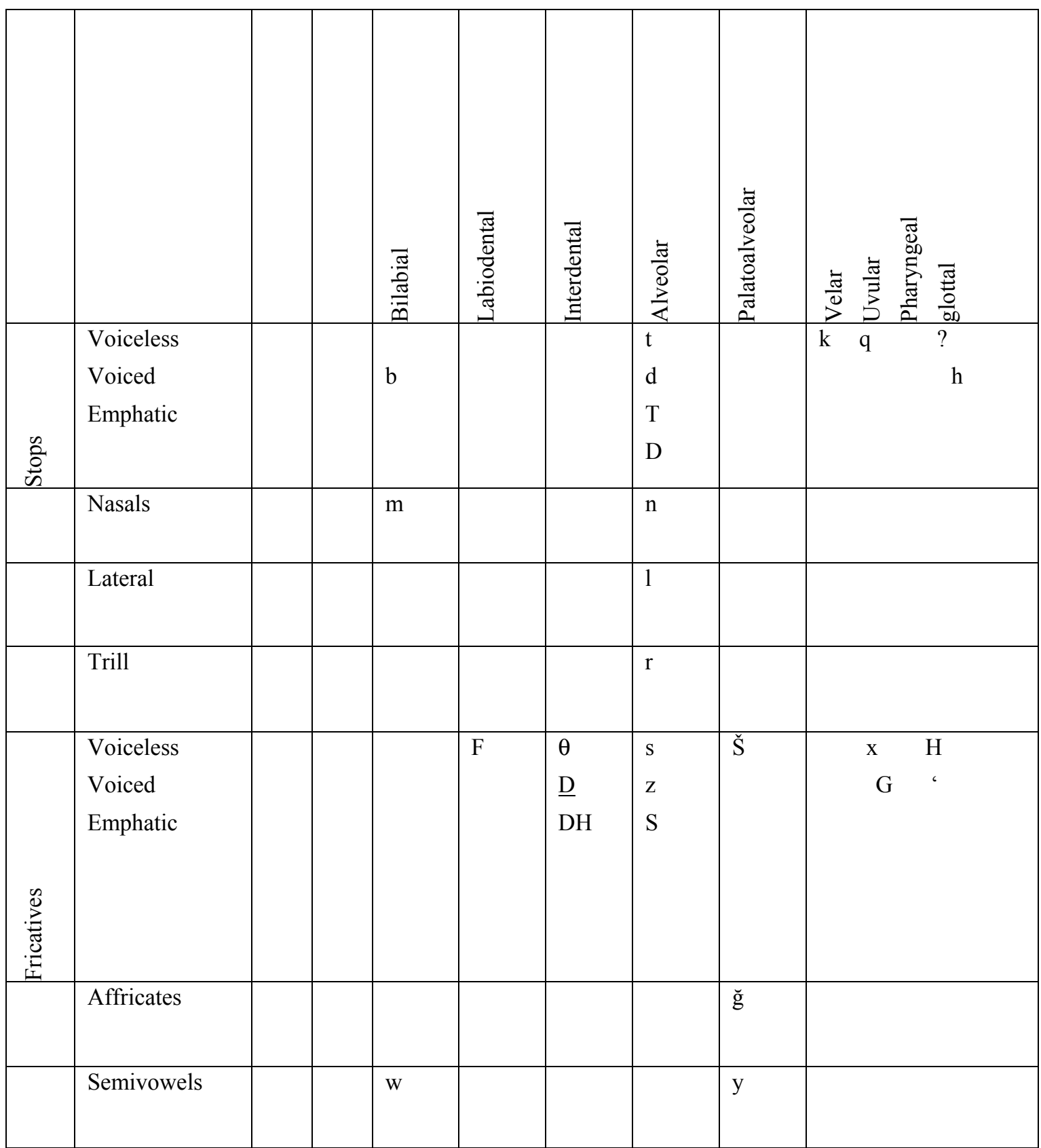

Table 3. Phonemic Inventory of Arabic Consonants 


\subsection{Classification of Consonant in Arabic:}

Phonetically, the Arabic consonants are classified on the basis of points of articulation and manners of articulation. In the former case, the points of contact of the active and passive articulators are taken into consideration. Following this mode, the consonants of Arabic are classified into bilabial, labiodentel, inter-dental, alveolar, palato-alveolar, velar, uvular, glottal and pharyngeal categories. It needs to be mentioned that in some Arabic consonants velarization accompanies their primary mode of articulation.

On the basis of manner of articulation, the consonants are classified following the mode or way in which they are articulated. In terms of this classification the consonants of Arabic are categorized as stops, nasals, affricates, fricatives, laterals, trills and semivowels. The feature of voicing is also present in Arabic and both voiced and voiceless consonants are found in Arabic language.

\subsection{Description of Consonant in Arabic:}

This section deals with the description of the consonants of Arabic. The description presents the characteristics of every consonant and the positions they occupy in different words. Here, every consonant is separately described.

\section{Stops:}

Arabic has nine stops whose description is provided below:

$/ \mathrm{b} /$

/b/: is a bilabial voiced stop. It occurs in all the three positions, i.e., initial, medial and final, of a word e.g.

1. Initial Position:

\begin{tabular}{|l|l|}
\hline Arabic Example & English Meaning \\
\hline bašar & 'human beings' \\
\hline baHr & 'Sea' \\
\hline bayt/bait & 'home' \\
\hline bali:G & 'eloquent' \\
\hline ba'd & 'afterwards' \\
\hline basi:T & 'simple' \\
\hline burha:n & 'proof' \\
\hline baxt 'luck & 'good fortune' \\
\hline
\end{tabular}

Table 1

2. Medial Position:

\begin{tabular}{|l|l|}
\hline Arabic Example & English Meaning \\
\hline 'abada & 'to worship' \\
\hline HaSba: & 'average type of pearl' \\
\hline miSba:H & 'lamp' \\
\hline muba:HaOa & 'discussion' \\
\hline abda:n & 'body (pl.)' \\
\hline maba:rid & 'files (instrument)' \\
\hline muba:rak - & 'blessed' \\
\hline
\end{tabular}

Table 2 
3. Final Position:

\begin{tabular}{|l|lc|}
\hline Arabic Example & English Meaning & 'dog' \\
\hline kalb & & 'liar' \\
\hline la'ab & 'play' & 'heart' \\
\hline ka:Dib & & 'book' \\
\hline bawwa:b & 'door-keeper' & \\
\hline qalib & & \\
\hline kita:b & 'dust' \\
\hline tura:b & \multicolumn{2}{|l}{} \\
\hline
\end{tabular}

Table 3

$/ \mathrm{t} /$

$/ t /$ : It is a voiceless alveolar stop. It is also found in all the three positions of a word e.g.

1. Initial Position:

\begin{tabular}{|l|l|}
\hline Arabic Example & English Meaning \\
\hline tamr & 'dates' \\
\hline tura:b & 'dust' \\
\hline ti:nun & 'figs' \\
\hline tuHfa & 'precious article, \\
\hline tarğama & 'translation' \\
\hline tisa' & 'nine' \\
\hline tuffa:H & 'apple' \\
\hline tau:t & 'mulberry' \\
\hline ta: $\breve{g}$ & 'crown' \\
\hline
\end{tabular}

Table 1

2. Medial Position:

\begin{tabular}{|l|l|}
\hline Arabic Example & English Meaning \\
\hline Kita:b & 'book' \\
\hline ixtira:' & 'invention' \\
\hline muxt:ar & 'selection, village headman' \\
\hline kita:b & 'book' \\
\hline mutarğim & 'translator, \\
\hline tamtama & 'to stammer' \\
\hline ixtila:f & 'difference' \\
\hline dustu:r & 'rule, regulation political constitution \\
\hline
\end{tabular}

Table 2 
3. Final Position:

\begin{tabular}{|l|l|}
\hline Arabic Example & English Meaning \\
\hline suku:t & 'silence' \\
\hline maxlu:qa:t & 'created things' \\
\hline baxt & 'luck' \\
\hline tu:t & 'mulberry' \\
\hline bayt/bait & 'home' \\
\hline baxi:t & 'lucky, fortunate (pl.)' \\
\hline Ha:nu:t & 'shop, wine shop' \\
\hline zayt/zait & 'oil' \\
\hline sabt & 'Sabbath, Saturday' \\
\hline
\end{tabular}

Table 3

$/ \mathrm{d} /$

/d/: It is a voiced, alveolar stop and its distribution is exemplified through the following examples:

1. Initial Position:

\begin{tabular}{|l|l|}
\hline Arabic Example & English Meaning \\
\hline darasa & 'to study' \\
\hline dunya: & 'world' \\
\hline dali: 1 & 'guide' \\
\hline
\end{tabular}

Table 1

2. Medial Position:

\begin{tabular}{|l|l|}
\hline Arabic Example & English Meaning \\
\hline madras & 'school' \\
\hline Mudi:r & 'director, manager, governor' \\
\hline bidu:n & 'without' \\
\hline abda:n & 'body (pl.)' \\
\hline miqda:r & 'quantety' \\
\hline
\end{tabular}

Table 2

3. Final Position:

\begin{tabular}{|l|l|}
\hline Arabic Example & English Meaning \\
\hline Hadid & 'iron' \\
\hline yad & 'hand \\
\hline ba'i:d & 'far' \\
\hline ba'd & 'afterwards' \\
\hline mabarid & 'files (instrument)' \\
\hline
\end{tabular}

Table 3

/T/

/T/: It is a voiceless emphatic alveolar stop and occupies all the three positions in a word e.g. 
1. Initial Position:

\begin{tabular}{|l|l|}
\hline Arabic Example & English Meaning \\
\hline Tabi:b & 'physician' \\
\hline Ta'a:m & 'food' \\
\hline Tifl & 'child, baby' \\
\hline Tawi: 1 & 'long, tall' \\
\hline
\end{tabular}

Table 1

2. Medial Position:

\begin{tabular}{|l|l|}
\hline Arabic Example & English Meaning \\
\hline ba:Til & 'vain, useless' \\
\hline maT'am & 'restaurant' \\
\hline ba:Tin & 'interior, hidden' \\
\hline
\end{tabular}

Table 2

3. Final Position:

\begin{tabular}{|l|l|}
\hline Arabic Example & English Meaning \\
\hline GalaT & 'wrong' \\
\hline mabsu:T & 'contented, happy, cheerful' \\
\hline basi:T & 'simple' \\
\hline
\end{tabular}

Table 3

$/ \mathrm{D} /$

/D/: is a voiced emphatic alveolar stop. This phoneme is present in all the three positions, which is elucidated through the given examples.

1. Initial Position:

\begin{tabular}{|l|l|}
\hline Arabic Example & English Meaning \\
\hline Daru:ri & 'necessary, essential' \\
\hline Da'i:f & 'weak' \\
\hline
\end{tabular}

Table 1

2. Medial Position:

\begin{tabular}{|l|l|}
\hline Arabic Example & English Meaning \\
\hline 'aXDar & 'green' \\
\hline maDbu:T ' & correct, right, well regulated' \\
\hline
\end{tabular}

Table 2

3. Final Position:

\begin{tabular}{|l|l|}
\hline Arabic Example & English Meaning \\
\hline 'abyaD & 'white' \\
\hline 'ari:D & 'wide' \\
\hline mari:D & 'ill, sick' \\
\hline
\end{tabular}

Table 3

$/ \mathrm{k} /$

$/ \mathrm{k} /$ : A voiceless velar stop, the phoneme $/ \mathrm{K} /$ is found in all the three positions, i.e., initial medial and final of a word. Its distribution is given below: 
1. Initial Position:

\begin{tabular}{|l|l|}
\hline Arabic Example & English Meaning \\
\hline kabi:r & 'great, big' \\
\hline kala:m & 'speech' \\
\hline kita:b & 'book' \\
\hline ka:Dib & 'liar' \\
\hline
\end{tabular}

Table 1

2. Medial Position:

\begin{tabular}{|l|l|}
\hline Arabic Example & English Meaning \\
\hline maktu:b & 'letter' \\
\hline mutakalim & 'speaker' \\
\hline maktabah & 'library' \\
\hline
\end{tabular}

Table 2

3. Final Position:

\begin{tabular}{|l|l|}
\hline Arabic Example & English Meaning \\
\hline malik & 'king' \\
\hline muba:rak & 'blessed' \\
\hline DaHak & 'laugh (v.)' \\
\hline šawk & 'thorns' \\
\hline
\end{tabular}

Table 3

$/ \mathrm{q} /$

/q/: It is a voiceless, uvular stop which is found in initial, medial and final positions of a word. For example,

1. Initial Position:

\begin{tabular}{|l|l|}
\hline Arabic Example & English Meaning \\
\hline qalb & 'heart' \\
\hline qabr & 'cemetery' \\
\hline
\end{tabular}

Table 1

2. Medial Position:

\begin{tabular}{|l|l|}
\hline Arabic Example & English Meaning \\
\hline miqda:r & 'amount' \\
\hline taqri:r & 'report' \\
\hline mustaqi:m & 'straight' \\
\hline maxlu:qa:t & 'created things' \\
\hline
\end{tabular}

Table 2

3. Final Position:

\begin{tabular}{|l|l|}
\hline Arabic Example & English Meaning \\
\hline qalaq & 'trouble' \\
\hline na:Tiq & 'spokesman, speaker' \\
\hline manTiq & 'logic' \\
\hline
\end{tabular}

Table 3 
$1 \%$

$/ /$ : It is a voiceless glottal stop. Its distribution is illustrated in the below given examples.

1. Initial Position :

\begin{tabular}{|l|l|}
\hline Arabic Example & English Meaning \\
\hline 'inkasar & 'to broken' \\
\hline 'aHmar & 'red' \\
\hline 'aXDar & 'green' \\
\hline 'aswad & 'black' \\
\hline
\end{tabular}

Table 1

2. Medial Position:

\begin{tabular}{|l|l|}
\hline Arabic Example & English Meaning \\
\hline qara'a & 'to read' \\
\hline li'anna & 'because' \\
\hline ta' $\theta \mathrm{i}: \mathrm{r}$ & 'influence impression' \\
\hline ta'abbaTa & 'to carry under arms' \\
\hline
\end{tabular}

Table 2

3. Final Position:

\begin{tabular}{|l|l|}
\hline Arabic Example & English Meaning \\
\hline buka:' & 'weeping' \\
\hline somaa' & 'sky' \\
\hline xabba' & 'to conceal, hide' \\
\hline
\end{tabular}

Table 3

$/ \mathrm{h} /$

$/ \mathrm{h} / \mathrm{I}$ It is a voiced glottal stop. The distribution of this phoneme is a s follows:

1. Initial Position:

\begin{tabular}{|l|l|}
\hline Arabic Example & English Meaning \\
\hline hal & 'state, or condition' \\
\hline ha:Da & 'this' \\
\hline hum & 'they' \\
\hline huwa & 'he' \\
\hline
\end{tabular}

Table 1

2. Medial Position:

\begin{tabular}{|l|l|}
\hline Arabic Example & English Meaning \\
\hline mahram & 'male escort' \\
\hline zahra & 'flower' \\
\hline gabha & 'forehead' \\
\hline za:hir & 'shining, flourishing \\
\hline sahl & 'easy' \\
\hline
\end{tabular}

Table 2 
3. Final Position:

\begin{tabular}{|l|l|}
\hline Arabic Example & English Meaning \\
\hline ru:h & 'soul' \\
\hline wiğh & 'face' \\
\hline SaHa:fah & 'press, journalism \\
\hline hiğrah & 'migration' \\
\hline
\end{tabular}

Table 3

\section{Affricates:}

Arabic has only one affricate, namely, $\breve{g}$. It needs to be mentioned that some scholars have classified it as a fricative. However, its pronunciation by majority of the native speakers favorite, its being an affricate. It is found in all the three positions of a word.

1. Initial Position:

\begin{tabular}{|l|l|}
\hline Arabic Example & English Meaning \\
\hline ğabba:r & 'mighty' \\
\hline ğabi:n & 'forehead, brow' \\
\hline ğadi:d & 'new' \\
\hline
\end{tabular}

Table 1

2. Medial Position:

\begin{tabular}{|l|l|}
\hline Arabic Example & English Meaning \\
\hline Hağar & 'stone' \\
\hline wa: ̆̆id & 'many' \\
\hline wiğh & 'face' \\
\hline
\end{tabular}

Table 2

3. Final Position:

\begin{tabular}{|l|l|}
\hline Arabic Example & English Meaning \\
\hline Hağ $\breve{g}$ & 'Pilgrimage' ( to Mecca) \\
\hline$\theta$ al̆ & 'snow' \\
\hline zauğ/zaw $\breve{g}$ & 'husband' \\
\hline
\end{tabular}

Table 3

\section{Fricatives:}

In a fricative sound the points of articulation come very close to each other so that the air-stream passes through them with a friction. There are twelve fricatives in Arabic whose description follows:

/f/

/f/: It is a voiceless labio-dental fricative and occupies all the initial, medial and final positions of a word. For example:

1. Initial Position:

\begin{tabular}{|l|l|}
\hline Arabic Example & English Meaning \\
\hline fakkar & 'think' \\
\hline fard & 'duty', religious duty' \\
\hline fa:kiha & 'fruit' \\
\hline faras & 'horse' \\
\hline fari:q & 'team, division' \\
\hline faSi:H & 'elequent' \\
\hline
\end{tabular}

Table 1 
2. Medial Position:

\begin{tabular}{|l|l|}
\hline Arabic Example & English Meaning \\
\hline daf' & 'push' \\
\hline rafD & 'refusal' \\
\hline nafar & 'person' \\
\hline tanaffas & 'breathe' \\
\hline šifa:h & 'lips' \\
\hline tafsi:r & 'exegesis' \\
\hline 'aSfar & 'yellow' \\
\hline
\end{tabular}

Table 2

3. Final Position:

\begin{tabular}{|l|l|}
\hline Arabic Example & English Meaning \\
\hline nahi:f & 'thin' \\
\hline xafi:f & 'thin' \\
\hline xaTaf' & to set sail' \\
\hline 'inSa:f & 'justice, equality, impartiality' \\
\hline niSf & 'half' \\
\hline
\end{tabular}

Table 3

$/ \theta /$

$/ \theta /$ : It is a voiceless interdental fricative and has the below given distribution.

1. Initial Position:

\begin{tabular}{|l|l|}
\hline Arabic Example & English Meaning \\
\hline$\theta$ aqi: 1 & 'heavy' \\
\hline$\theta$ ala: $\theta$ a & 'three' \\
\hline$\theta a: n i$ & 'other' \\
\hline$\theta a: b i t$ & 'firm, sure' established' \\
\hline
\end{tabular}

Table 1

2. Medial Position:

\begin{tabular}{|l|l|}
\hline Arabic Example & English Meaning \\
\hline$\theta$ ala $\theta \mathrm{a}$ & 'three' \\
\hline mu$\theta$ alla $\theta$ & 'triangle' \\
\hline
\end{tabular}

Table 2

3. Final Position:

\begin{tabular}{|l|l|}
\hline Arabic Example & English Meaning \\
\hline$\theta a: l i \theta$ & 'third' \\
\hline mu $\theta$ alla $\theta$ & 'triangle' \\
\hline
\end{tabular}

Table 3

/s/

/s/: It is a voiceless alveolar fricative and has the following distribution. For example: 
1. Initial Position:

\begin{tabular}{|l|l|}
\hline Arabic Example & English Meaning \\
\hline sinn & 'tooth' \\
\hline saHa:b & 'raincloud' \\
\hline sabi: 1 & 'path, way, \\
\hline sa:biq & 'former, previous, forerunner \\
\hline
\end{tabular}

Table 1

2. Medial Position:

\begin{tabular}{|l|l|}
\hline Arabic Example & English Meaning \\
\hline mustaqi:m & 'straight' \\
\hline xamsa & 'five' \\
\hline 'aswad & 'black' \\
\hline lisa:n & 'tongue' \\
\hline masru:r & 'glad' \\
\hline
\end{tabular}

Table 2

3. Final Position:

\begin{tabular}{|l|l|}
\hline Arabic Example & English Meaning \\
\hline šams & 'sun' \\
\hline ra:s & 'head' \\
\hline firdaus & 'paradise, garden' \\
\hline muflis & 'bankrupt, poor' \\
\hline
\end{tabular}

Table 3

$\mathrm{S} /$

/S/: It is an emphatic alveolar fricative. The /distribution of this phoneme in various positions is given below:

1. Initial Position:

\begin{tabular}{|l|l|}
\hline Arabic Example & English Meaning \\
\hline Sa:liH & 'good, proper' \\
\hline Sa:mit & 'silent' \\
\hline Sa:yim & 'one who fasts' \\
\hline Sira:T & 'way, path' \\
\hline
\end{tabular}

Table 1

2. Medial Position:

\begin{tabular}{|l|l|}
\hline Arabic Example & English Meaning \\
\hline iSTila:H & 'technical use, idiom' \\
\hline iSla:H & 'reform, movement' \\
\hline taSwi:r & 'picture' \\
\hline 'Sfar & 'yellow' \\
\hline
\end{tabular}

Table 2 
3. Final Position:

\begin{tabular}{|l|l|}
\hline Arabic Example & English Meaning \\
\hline mulaxxaS & 'summary, abstract' \\
\hline muxliS & 'faithful' \\
\hline qaSS & 'to cut' \\
\hline
\end{tabular}

Table 3

$/ \check{\mathbf{S}} /$

/̌s/: It is a voiceless alveopalatal fricative and its distribution is provided below:

1. Initial Position:

\begin{tabular}{|l|l|}
\hline Arabic Example & English Meaning \\
\hline šarab & 'drink' \\
\hline šiğara & 'tree' \\
\hline šahi:d & 'martyr' \\
\hline ša:hid & 'witness' \\
\hline
\end{tabular}

Table 1

2. Medial Position:

\begin{tabular}{|l|l|}
\hline Arabic Example & English Meaning \\
\hline Haši:š & 'grass' \\
\hline raši:d & 'upright, righteous' \\
\hline raši:q & 'fine, elegant' \\
\hline mašhu:r & 'famous' \\
\hline
\end{tabular}

Table 2

3. Final Position:

\begin{tabular}{|l|l|}
\hline Arabic Example & English Meaning \\
\hline Haši:š & 'grass' \\
\hline mun'iš & 'refreshing' \\
\hline 'af̌s & 'luggage' \\
\hline
\end{tabular}

Table 3

$/ \mathrm{x} /$

/ $\mathrm{x} /:$ It is a voiceless uvular fricative and occurs in initial, medial and final positions. For example:

1. Initial Position:

\begin{tabular}{|l|l|}
\hline Arabic Example & English Meaning \\
\hline xadd & 'cheek' \\
\hline xa:lah & 'aunt' \\
\hline xa: 1 & 'uncle' \\
\hline xabi: $r$ & expert, well informed' \\
\hline xabar & 'news' \\
\hline xa:dim & 'servant' \\
\hline
\end{tabular}

Table 1 
2. Medial Position:

\begin{tabular}{|l|l|}
\hline Arabic Example & English Meaning \\
\hline naxal & 'to sieve' \\
\hline 'uxt & 'sister' \\
\hline 'axba:r & 'news (pl.)' \\
\hline maxrağ & 'outlet, issue, exit' \\
\hline
\end{tabular}

Table 2

3. Final Position:

\begin{tabular}{|l|l|}
\hline Arabic Example & English Meaning \\
\hline Tabax & 'to cook' \\
\hline maTbax & 'kitchen' \\
\hline 'ax & 'brother' \\
\hline
\end{tabular}

Table 3

/H/

/H/: It is a voiceless pharyngeal fricative and its phonetic distribution is as follows:

1. Initial Position:

\begin{tabular}{|l|l|}
\hline Arabic Example & English Meaning \\
\hline Hala:l & 'lawful, right' \\
\hline Hali:b & 'milk' \\
\hline Ha:kim & 'ruler' \\
\hline Haki:m & 'wise (man), doctor' \\
\hline
\end{tabular}

Table 1

2. Medial Position:

\begin{tabular}{|l|l|}
\hline Arabic Example & English Meaning \\
\hline miHra:b & 'niche in mosque, direction of Mecca' \\
\hline maHbu:b & 'beloved \\
\hline 'aHba:r & 'learned man, doctor' \\
\hline maHdadih & 'blacksmith place' \\
\hline laHm & 'meet, flesh' \\
\hline
\end{tabular}

Table 2

3. Final Position:

\begin{tabular}{|l|l|}
\hline Arabic Example & English Meaning \\
\hline ri:H & 'wind' \\
\hline ru:H & 'soul, spirit' \\
\hline $\mathrm{Ta}: \mathrm{H}$ & 'fall' \\
\hline $\mathrm{SaHH}$ & 'right, correct' \\
\hline milH & 'salt' \\
\hline masaH & 'wipe' \\
\hline
\end{tabular}

Table 3

/D/

/D/: It is a voiced interdental fricative and is found in initial, medial and final positions. For example: 
1. Initial Position:

\begin{tabular}{|l|l|}
\hline Arabic Example & English Meaning \\
\hline$\underline{\text { Dahabi }}$ & 'gold' \\
\hline$\underline{\text { Dira' }}$ & 'arm' \\
\hline$\underline{\text { Duba:b }}$ & 'flies' \\
\hline$\underline{\text { DabaH }}$ & 'to slay, slaughter' \\
\hline$\underline{\text { Dali:l }}$ & 'low, abject, wretched' \\
\hline
\end{tabular}

Table 1

2. Medial Position:

\begin{tabular}{|c|c|}
\hline Arabic Example & English Meaning \\
\hline ği $\underline{D}^{\prime}$, & 'palm trunk' \\
\hline iDin & 'ear' \\
\hline $\mathrm{kaDab}$ & 'tell untruth' \\
\hline ha: $\underline{\mathrm{Da}}$ & 'this' \\
\hline
\end{tabular}

Table 2

3. Final Position:

\begin{tabular}{|l|l|}
\hline Arabic Example & English Meaning \\
\hline tilmi: $\underline{\mathrm{D}}$ & 'pupil' \\
\hline La무묘 & 'delicious' \\
\hline
\end{tabular}

Table 3

/DH/

/DH/: It is an emphatic interdental fricative and has the below given distribution.

1. Initial Position:

\begin{tabular}{|l|l|}
\hline Arabic Example & English Meaning \\
\hline DHa:hir & 'manifest, external' \\
\hline DHifir & 'nail' \\
\hline DHahr & 'back' \\
\hline DHari:f & 'witty person' \\
\hline DHa:lim & 'oppressor' \\
\hline
\end{tabular}

Table 1

2. Medial Position:

\begin{tabular}{|l|l|}
\hline Arabic Example & English Meaning \\
\hline 'aDhm & 'bone' \\
\hline muDHa:harah & 'demonstration' \\
\hline maDHlu:m & 'oppressed, having a grievance' \\
\hline muDHlim & 'dark' \\
\hline 'aDHhar & 'to show' \\
\hline
\end{tabular}

Table 2 
3. Final Position:

\begin{tabular}{|l|l|}
\hline Arabic Example & English Meaning \\
\hline Gali:DH & 'thick' \\
\hline lafDH & 'word' \\
\hline La:HaDH & 'to note' \\
\hline HaafiDH & 'keeper; memorizer' \\
\hline
\end{tabular}

/z/

/z/: It is voiced alveolar fricative. It occurs in below given distribution.

1. Initial Position:

\begin{tabular}{|c|c|}
\hline Arabic Example & English Meaning \\
\hline zawğa & 'wife' \\
\hline zo: $\breve{g}$ & 'husband' \\
\hline zabu:n & 'customer' \\
\hline zuğa: ̆g & 'glass' \\
\hline zaka:h & 'alms in Islam' \\
\hline zaman & 'time' \\
\hline ziya:rah & 'a visit' \\
\hline zaitu:n & 'olives' \\
\hline
\end{tabular}

Table 1

2. Medial Position:

\begin{tabular}{|l|l|}
\hline Arabic Example & English Meaning \\
\hline 'azraq & 'blue' \\
\hline muz'iğ & 'disturbing, upsetting' \\
\hline 'izdiHa:m & 'crowding, a crowd' \\
\hline zalazalah & 'earthquake' \\
\hline 'iğa:zah & 'permission, licence, leave' \\
\hline Huzn & 'sadness' \\
\hline Hazi:n & 'sad' \\
\hline Hizb & 'party' \\
\hline
\end{tabular}

Table 2

3. Final Position:

\begin{tabular}{|l|l|}
\hline Arabic Example & English Meaning \\
\hline 'aruz & 'rice' \\
\hline ba:riz & 'prominent, outstanding' \\
\hline ğa'iz & 'passing, lawful' \\
\hline 'a:ğiz & 'helpless, unable, Impotent' \\
\hline
\end{tabular}

Table 3

/G/

$/ \mathrm{G} /$ : is a voiced uvular fricative and has the below given distribution 
1. Initial Position:

\begin{tabular}{|l|l|}
\hline Arabic Example & English Meaning \\
\hline Gala & 'to boil' \\
\hline GawwaaS & 'diver' \\
\hline Guba:r & 'dust' \\
\hline Ganna & 'Sing' \\
\hline Gali:DH & 'thick' \\
\hline Gasal & 'to wash' \\
\hline
\end{tabular}

Table 1

2. Medial Position:

\begin{tabular}{|l|l|}
\hline Arabic Example & English Meaning \\
\hline maGrib & 'west' \\
\hline maGfirah & 'forgiveness, \\
\hline 'aGla:T & 'mistake' (pl.) \\
\hline maGmu:m & 'anxious, troubled, grieved' \\
\hline
\end{tabular}

Table 2

3. Final Position:

\begin{tabular}{|l|l|}
\hline Arabic Example & English Meaning \\
\hline marsaG & 'wrist' \\
\hline bali:G & 'eloquent' \\
\hline mablaG & 'sum (of money), amount' \\
\hline
\end{tabular}

Table 3

16

$/ \%$ : It is a voiced pharyngeal fricative and occurs in the initial, medial and final positions. For example:

1. Initial Position

\begin{tabular}{|l|l|}
\hline Arabic Example & English Meaning \\
\hline 'amm & 'uncle' \\
\hline 'amma & 'aunt' \\
\hline 'ayn & 'eye' \\
\hline 'ind & 'at' \\
\hline 'aDD & 'bite(v)' \\
\hline 'aDHm & 'bone' \\
\hline 'ari:D & 'wide' \\
\hline
\end{tabular}

Table 1

2. Medial Position:

\begin{tabular}{|l|l|}
\hline Arabic Example & English Meaning \\
\hline ša'ar & 'hair' \\
\hline ka'b & 'heel' \\
\hline am'a & 'guts' \\
\hline qa'ad & 'sit' \\
\hline na:'im & 'smooth' \\
\hline ta'li:m & 'education, instruction' \\
\hline mu'allim & 'teacher' \\
\hline
\end{tabular}

Table 2 
3. Final Position:

\begin{tabular}{|l|l|}
\hline Arabic Example & English Meaning \\
\hline iSba & 'finger' \\
\hline qaTa'a & 'cut (v.)' \\
\hline sama'a & 'hear' \\
\hline${\text { bi }{ }^{`}}^{`}$ & 'some (number between 3 and 10) ' \\
\hline maSna $^{6}$ & 'factory' \\
\hline
\end{tabular}

Table 3

Nasals:

There are two nasals i.e. $/ \mathrm{m} /$ and $/ \mathrm{n} /$ whose description is provided below:

$/ \mathrm{m} /$

$/ \mathrm{m} /$ : It is a bilabial nasal and has the below given distribution.

1. Initial Position:

\begin{tabular}{|l|l|}
\hline Arabic Example & English Meaning \\
\hline maSna' & 'factory' \\
\hline madrasah & 'school' \\
\hline maktab & 'office' \\
\hline maTbax & 'kitchen, bakery' \\
\hline munkar & 'abmoination' \\
\hline maktabah & 'library' \\
\hline
\end{tabular}

Table 1

1. Medial Position:

\begin{tabular}{|l|l|}
\hline Arabic Example & English Meaning \\
\hline 'aHmar & 'red' \\
\hline xamsah & 'five' \\
\hline ğamada & 'to free(v) ' \\
\hline sama'a & 'hear' \\
\hline qamar & 'moon' \\
\hline šams & 'sun' \\
\hline
\end{tabular}

Table 2

2. Final Position:

\begin{tabular}{|l|l|}
\hline Arabic Example & English Meaning \\
\hline 'umm & 'mother' \\
\hline 'amm & uncle' \\
\hline Ham & father-in-law' \\
\hline damm & blood' \\
\hline laHm & meat' \\
\hline mustaqi:m & 'straight' \\
\hline
\end{tabular}

Table 3 
$/ \mathrm{n} /$

/n/: It is an alveolar nasal. Its distribution in initial, medial and final positions is exemplified below:

1. Initial Position:

\begin{tabular}{|l|l|}
\hline Arabic Example & English Meaning \\
\hline na:r & 'fire' \\
\hline naxlah & 'female \\
& date palm' \\
\hline na:dir, & 'rare, rare thing, \\
\hline nisa'? & 'women' \\
\hline na:SiH & 'adviser' \\
\hline
\end{tabular}

Table 1

2. Medial Position:

\begin{tabular}{|l|l|}
\hline Arabic Example & English Meaning \\
\hline Sanah & 'year' \\
\hline 'iOnayn & 'two' \\
\hline Ganna & 'sing' \\
\hline Өa:ni & 'other' or 'second' \\
\hline manSu:r & 'conqueror (lit. the assisted of God' \\
\hline 'inSa:f & 'equity, justice, impartiality' \\
\hline
\end{tabular}

Table 2

3. Final Position:

\begin{tabular}{|l|l|}
\hline Arabic Example & English Meaning \\
\hline man & 'who' \\
\hline 'iOnayn & 'two' \\
\hline lisa:n & 'tongue' \\
\hline duxxa:n & 'smoke' \\
\hline
\end{tabular}

Table 3

Lateral:

Arabic possesses a single alveolar lateral, i.e., /I/ which occupies the initial, medial and final positions. For example:

1. Initial Position:

\begin{tabular}{|l|l|}
\hline Arabic Example & English Meaning \\
\hline lisa:n & 'tongue' \\
\hline layl & 'night' \\
\hline la'iba & 'to play' \\
\hline la:zim & 'necessary' \\
\hline laㅁi: $\underline{D}$ & 'sweet' or 'delightful' \\
\hline
\end{tabular}

Table 1 
2. Medial Position:

\begin{tabular}{|l|l|}
\hline Arabic Example & English Meaning \\
\hline kalb & 'dog' \\
\hline qalb & 'heart' \\
\hline$\theta$ al̆ & 'ice' \\
\hline$\theta$ ala: $\theta$ ah & 'three' \\
\hline 'alfa:DH & 'words' \\
\hline alqa:b & 'surnames, titles, epithets' \\
\hline
\end{tabular}

Table 2

3. Final Position:

\begin{tabular}{|l|l|}
\hline Arabic Example & English Meaning \\
\hline 'akala & 'eat' \\
\hline qali:l & 'few' or 'little' \\
\hline Өaqi: & 'heavy' \\
\hline Tawi:1 & 'long', or 'tall' \\
\hline qa:la & 'to say' \\
\hline
\end{tabular}

Table 3

Trill:

A single trill, i.e., alveolar $/ \mathrm{r} /$ is present in Arabic whose distribution follows:

1. Initial Position:

\begin{tabular}{|l|l|}
\hline Arabic Example & English Meaning \\
\hline ra'i:s & 'head, chairman, chief \\
\hline rabi:' & 'spring' \\
\hline rajab & 'seventh month of Islamic calender' \\
\hline riHlah & 'journey' \\
\hline rağul & 'man' \\
\hline ra's & 'head' \\
\hline
\end{tabular}

Table 1

2. Medial Position:

\begin{tabular}{|l|l|}
\hline Arabic Example & English Meaning \\
\hline tarHa:b & 'welcome' \\
\hline mura:sil & 'newspaper correspondent' \\
\hline mura:qib & 'supervisor, foreman, controller' \\
\hline 'arwa:H & 'soul (pl.),spirit (pl.) ' \\
\hline 'azraq & 'blue' \\
\hline
\end{tabular}

Table 2 
3. Final Position:

\begin{tabular}{|l|l|}
\hline Arabic Example & English Meaning \\
\hline 'azra:r & 'button (pl.)' \\
\hline za:'ir & ' visitor' \\
\hline zahr & ' flower, blossom' \\
\hline za:hir ' & 'shining, flourishing \\
\hline sirr & ' secret' $^{\prime}$ \\
\hline 'axDar & ' green' $^{\prime}$ \\
\hline DHahr & ' back' \\
\hline qamar & ' moon' $^{\prime}$ \\
\hline
\end{tabular}

Table 3

\section{Semivowels:}

Arabic language possesses two semivowels which are described in the following lines,

$/ \mathrm{w} /$

/w/: It is a bilabial semivowel which occurs in initial, medial and final positions. Its distribution is provided below:

1. Initial Position:

\begin{tabular}{|l|l|}
\hline Arabic Example & English Meaning \\
\hline waram & ' swell' \\
\hline wa:di & ' valley' \\
\hline waba:l & ' misfortune, evil \\
\hline wuӨu:q & ' trust, confidence' \\
\hline wa:ğib & ' a duty \\
\hline
\end{tabular}

Table 1

2. Medial Position:

\begin{tabular}{|l|l|}
\hline Arabic Example & English Meaning \\
\hline mudawwar & ' round' $^{\prime}$ \\
\hline ğawwa:1 & ' mobile' \\
\hline huwa & ' he' $^{\prime}$ \\
\hline awra:q & 'paper (pl.), \\
\hline awaza:n & ' weight, measure' \\
\hline
\end{tabular}

Table 2

3. Final Position:

\begin{tabular}{|l|l|}
\hline Arabic Example & English Meaning \\
\hline Daw' & ' light' \\
\hline musa:wa: & ' equalized' \\
\hline
\end{tabular}

Table 3

$/ \mathrm{Y} /$

/Y/: The other semivowel in Arabic is the alveopalatal /y/ whose distribution is provided below: 
1. Initial Position:

\begin{tabular}{|l|l|}
\hline Arabic Example & English Meaning \\
\hline yad & ' hand' \\
\hline ya'as & ' despair' \\
\hline yati:m & ' orphan' $^{\prime}$ \\
\hline yasi:r & ' easy, small' \\
\hline yaqi:n & ' certain belief, conviction' \\
\hline yami:n & ' right hand, oath' \\
\hline
\end{tabular}

Table 1

2. Medial Position:

\begin{tabular}{|l|l|}
\hline Arabic Example & English Meaning \\
\hline yaba:n & ' Japan' \\
\hline 'ayya:m & ' days' \\
\hline xayyaT & ' sew' \\
\hline 'abyaD & ' white' $^{\prime}$ \\
\hline
\end{tabular}

Table 2

3. Final Position:

\begin{tabular}{|l|l|}
\hline Arabic Example & English Meaning \\
\hline rama:diyy & ' grey' \\
\hline Hanafiyy & ' Hanafite, follower of the rite of Abu Hanifa' \\
\hline
\end{tabular}

Table 3

\subsection{Conclusion:}

The preceding pages provide an exhaustive description of the consonantal system of Arabic language.

The different consonants have been classified according to their points and manners of articulation and their distribution has been provided exhaustively. Therefore, it can be said that Arabic has a rich consonantal system.

\section{Phonemes / Allophones:}

Two phonetically dissimilar sounds, which occur in the same position and are contrastive, are called phonemes. Thus, $/ \mathrm{n} /$ and $/ \mathrm{m} / \mathrm{in} / \mathrm{nahr} /$ 'river' and /mahr/ 'dowry' are two different phonemes. A phoneme is realized differently, depending on its position in words. These positional defined realizations or variants are members of the same phoneme or its allophones. Thus, whereas $/ \mathrm{n} /$ and $/ \mathrm{m} /$ are phonemes in $/ \mathrm{nahr} / \mathrm{and} / \mathrm{mahr} /$ since the substitution of one for another gives a different word, $\{\mathrm{m}\}$ is an allophone of the phoneme $/ \mathrm{n} / \mathrm{in}$ (mamba') since the underlying form is $/ \mathrm{manba}^{\prime} /$ and $/ \mathrm{n} /$ is realized as $(\mathrm{m})$ under the effect of the phonetic assimilation. The pronunciation of the word either with $[\mathrm{n}]$ or $[\mathrm{m}]$ does no give a meaning change. It may only give an odd pronunciation in case of [n] in (manba').

The common allophones are as follows:
(i) Stops
(b) a voiced balabial unaspirated stop
$\left[\mathrm{k}^{\mathrm{h}}\right]$ a velar voiceless aspirated stop
[q] a uvular voiceless stop
$\left[\mathrm{t}^{\mathrm{h}}\right]$ a voiceless dental aspirated stop
[T] a voiceless dental valorised unaspirated stop
[d] a voiced dental unaspirated stop
[D] a voiced dental velarized unaspirated stop 
['] a voiced glottal stop

['] a voiced palato-alveolar affricate

['] Al-Ani (1971) writes "the /'/is described as a voiced pharyngeal fricative in all the previous works in Arabic, literally as well as dialects. However, after a thorough acoustical analysis the author has found that the common allophone of $/ \%$ is actually a voiceless stops and not a voiced fricative".

\section{(ii) Fricatives}

[f] a voiceless labio-dental fricative

$[\theta]$ a voiceless inter-dental fricative

[D] a voiced inter-dental fricative

[DH] a voiced velarized inter-dental fricative

[s] a voiceless sibilant alveolar fricative

[z] a voiced sibilant alveolar fricative $\mathrm{zx}$

[̌̌ a voiceless sibilant palato-alveolar fricative

[x] a voiceless velar fricative

$[\mathrm{H}]$ a voiceless velar fricative

[h] a voiceless oral fricative

(iii) Liquids

The phoneme /I/ has two distinct varieties: the emphatic (I) in which the front of the tongue makes contact with the alveolar ridge and the back of the tongue is raised towards the soft palate and the non-emphatic (f) in which the top of the tongue contacts, the front part of the alveolar ridge. The later is commoner than the former. The distinction between these two allophones can be clearly seen in the following example.

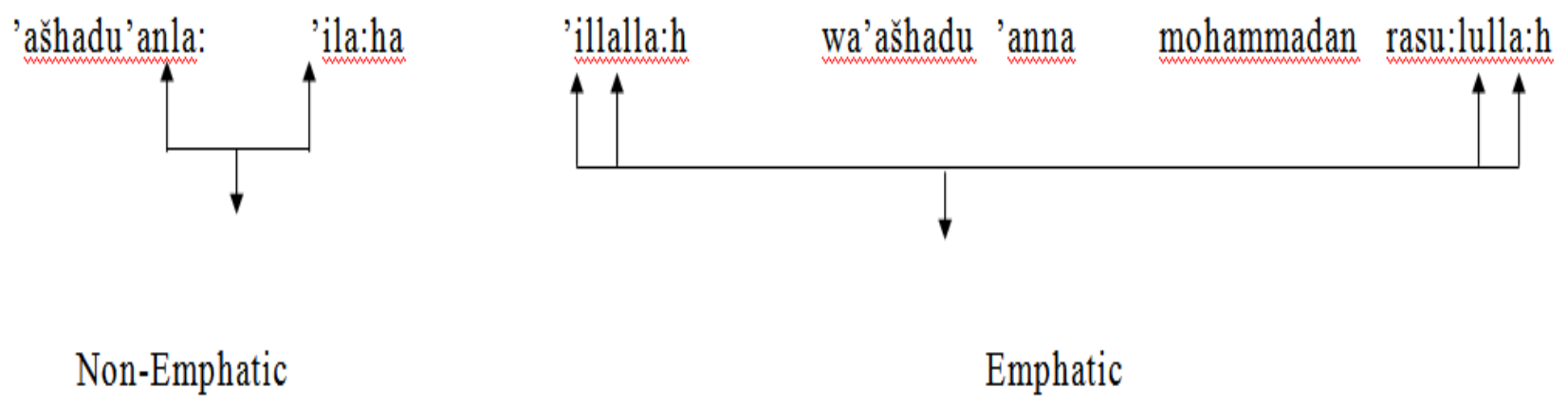

Figure (1)

'I bear witness that there is no God save? Allah and I bear witness that Mohammad is the prophet of 'alla:h'.

(iv) (r) is a voiced alveolar trill

(v)

Semi-vowels

(vi) (w) a voiced bilabial semi-vowel

(ii) $\quad[\mathrm{y}]$ a voiced palatal semi-vowel.

(vi) Nasals

[m] a voiced bilabial nasal.

The phoneme / $\mathrm{n} /$ has 4 allophones.

It is realised as labio-dental [m] when it is immediately followed by /b/ or /f/ as in [mamba'] 'spring' and [mamfu:x] 'scattered' 'blown up; swollen, pumped up. 
It is realized dental [n:] when it is immediately followed by the dentals $/ \theta /$ or $/ \underline{\mathrm{D}} /$ as in [man: $\theta u: r]$ 'scattered' 'prosaic' and [mun:Dir] 'warner' cautioner'.

It is realized as [D] when it is immediately followed by /k/ or /q/ as in ['iDkisa:r] 'state or process, of being broken, brokenness, breaking.

$/ \mathrm{n} /$ elsewhere

\subsection{Consonant clusters in Arabic}

There are no consonant clusters in word initial position. Clusters occur in medial and final positions. In medial position the cluster is broken up in pronunciation, the first consonant of the cluster being pronounced with the preceding vowel, the second with the following vowel. The medial clusters are clearly sequences of a syllable closing consonant and a syllable opening consonant. Samples of examples are given below:

(i)

Medial clusters:

\begin{tabular}{|l|l|l|}
\hline Symbol & Arabic Example & English Meaning \\
\hline$/ \mathrm{kt} /$ & Maktab & 'office' \\
\hline$/ \mathrm{br} /$ & Mibrad & 'file' \\
\hline$/ \mathrm{nx} /$ & Munxul & 'sieve' \\
\hline$/ ` 1 /$ & 'i'la:m & 'information' \\
\hline$/ \mathrm{nd} /$ & 'indafa'a & $\begin{array}{l}\text { 'to rushoff; to proceed without } \\
\text { forethought' }\end{array}$ \\
\hline$/ \mathrm{mt} /$ & 'imtaHana & 'to examine; subject to the test' \\
\hline$/ \mathrm{st} /, / \mathrm{xr} /$ & 'istaxrağa & 'toduce' \\
\hline
\end{tabular}

Table 1

\section{Final Clusters:}

\begin{tabular}{|l|l|l|}
\hline Symbol & Arabic Example & English Meaning \\
\hline$/ \mathrm{tl} /$ & Qatl & 'killing' \\
\hline$/ \mathrm{lb} /$ & Qalb & 'heart' \\
\hline$/ \mathrm{xl} /$ & Naxl & 'tree dates' pl. \\
\hline$/ \mathrm{rb} /$ & Darb & 'beating' \\
\hline$/ \mathrm{hb} /$ & nahb & robbery' \\
\hline$/ \mathrm{hr} /$ & $\mathrm{siHr}$ & 'sorcery' \\
\hline$/ \mathrm{hr} /$ & $\mathrm{Sihr}$ & 'son-in-law', 'brother-in-law' \\
\hline$/ \mathrm{lm} /$ & $\mathrm{Silm}$ & 'peace' \\
\hline$/ \mathrm{sm} /$ & Daxm & 'big', 'large', 'magnificent' \\
\hline$/ \mathrm{r} \theta /$ & Har $\theta$ & 'plowing', tilling' \\
\hline
\end{tabular}

Table 2

\section{Conclusion}

Arabic is one of the most prominent languages of the Hamitic branch of the Afro-Asiatic family also called the Hamito - Semitic family. It is infixing language in which word-formation involves predominantly infixing vowels in a root consisting entirely of consonants.

The preceding pages provide an exhaustive description of the consonantal system of Arabic language.

The different consonants have been classified according to their points and manners of articulation and their distribution has been provided exhaustively. Therefore, it can be said that Arabic has a rich consonantal system. 


\section{References}

Abboud, Peter. (1984). “Elementary Modern Standard Arabic”, Cambridge: Cambridge University Press.

Abercrombie, D. (1967). “Elements of General Phonetics”, Edinburgh; Edinburgh University Press.

Al-Ani, Salman, (1970). “Arabic Phonologyl” Mouton: The Hague. http://dx.doi.org/10.1515/9783110878769

Clements, G.N. (1991). "Place of Articulation in Consonants and Vowels", a united theory. Working Papers of the Cornell Phonetics Laboratory 5, 77-123.

Chejne, A. (1958). "The Role of Arabic in Present Day Arab Society", The Islamic Literature, 10, 4; $15-54$.

/Cowell, Mark W. (2005). "A Reference of Syrian Arabic With Audio CD" Printed in United States of America: Georgetown University Press.

Crothers, J. (1978). "Typology and Universals in Vowel Systems", In Greenberg (1978), Universal of Human Language. Stanford, Stanford University Press.

Karin, C. Ryding. (2006). A Reference Grammar of Modern Standard Arabic. Cambridge: UK, Cambridge University Press.

Fatihi, A.R. (2001). “Aspects of Arabic Phonology”. Delhi, Katinga Publication.

Greenberg, J. (ed.) (1978). “Universals of Human Language”, Stanford, Stanford University Press.

Haywood, J.A. \& H.M. Nahmad. (1965). “A new Arabic Grammar of the Written Language”. London, Lund Humphries.

Holes, Clive, (1990). “Gulf Arabic”, London and New York. Routledge.

Hualde, J.I. (1991). “Unspecified and Unmarked Vowels”, Linguistic Inquiry 22, 205-9.

Jones, Danid. (1965). “An Outline of English Phonetics”. ( $8^{\text {th }}$ Edition). Cambridge. Haffer.

Lindan, M. (1978). "Vowel Features". Language 54, 541-63 London. Oxford University Press. http://dx.doi.org/10.1353/lan.1978.0066

Wright, W. (1977). “A Grammar of the Arabic Language”. ( $3^{\text {rd }}$ Edition). Cambridge. Cambridge University Press. 